\title{
Inhaltsübersicht zu Band 2
}

Wichtiger Hinweis — $\mathrm{V}$

Vorwort — VII

VIII Elementare Streutheorie - 915

Übersicht über die Ergänzungen zu Kapitel VIII — 948

AVIII Freies Teilchen: Drehimpulseigenzustände - 949

B

$C_{\mathrm{VIII}} \quad$ Beispiele zur Streutheorie -969

IX Der Spin des Elektrons - 977

Übersicht über die Ergänzungen zu Kapitel IX — 992

A IX Drehoperatoren für ein Spin-1/2-Teilchen — 993

B $_{\mathrm{IX}} \quad$ Aufgaben $-\mathbf{1 0 0 0}$

X Addition von Drehimpulsen -1007

Übersicht über die Ergänzungen zu Kapitel X 1033

Ax Beispiele für die Addition von Drehimpulsen - 1034

BX Clebsch-Gordan-Koeffizienten — 1041

$C_{X} \quad$ Addition von Kugelflächenfunktionen - 1050

DX Das Wigner-Eckart-Theorem - 1055

EX Elektrische Multipolmomente - 1066

$F_{X} \quad$ Entwicklung gekoppelter Drehimpulse - 1080

GX Aufgaben -1095

XI Stationäre Störungstheorie -1103

Übersicht über die Ergänzungen zu Kapitel XI — 1117

AXI Gestörter harmonischer Oszillator - 1118

B XI $\quad$ Wechselwirkung zwischen magnetischen Dipolen - 1128

$C_{\mathrm{XI}} \quad$ Van-der-Waals-Kräfte -1139 
$D_{\mathrm{XI}} \quad$ Der Volumeneffekt -1151

EXI Die Variationsmethode -1158

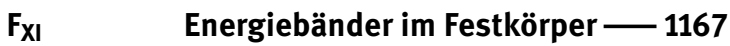

GXI Chemische Bindung: Das $\mathrm{H}_{2}^{+}$-Ion -1180

$\mathrm{H}_{\mathrm{XI}} \quad$ Aufgaben -1213

XII Fein- und Hyperfeinstruktur des Wasserstoffatoms - 1223

Übersicht über die Ergänzungen zu Kapitel XII — 1257

AxII Der Hyperfeinstruktur-Hamilton-Operator - 1258

BxII Erwartungswerte und Feinstruktur - 1267

$\mathrm{C}_{\mathrm{XII}} \quad$ Hyperfeinstruktur und Zeeman-Effekt für das Myonium und das Positronium — 1272

DXII Elektronenspin und Zeeman-Effekt - 1280

EXII Stark-Effekt des Wasserstoffatoms - 1289

XIII Näherungsmethoden für zeitabhängige Probleme - 1293

Übersicht über die Ergänzungen zu Kapitel XIII —1330

AxIII Atom und elektromagnetische Strahlung - 1331

BXIII Zweiniveausystem und sinusförmige Störung - 1350

C XsIII Oszillation zwischen zwei diskreten Zuständen bei einer sinusförmigen Störung — 1367

DxIII Zerfall eines diskreten Zustands in ein Kontinuum —1371

EXIII Stochastische zeitabhängige Störung. Relaxation — 1384

FXIII Aufgaben -1409

XIV Systeme identischer Teilchen -1421

Übersicht über die Ergänzungen zu Kapitel XIV — 1461

AxIV Mehrelektronenatome. Konfigurationen -1462

BXIV Energieniveaus des Heliumatoms - 1470

CXIV Elektronengas. Anwendung auf Festkörper - 1485

DxIV Aufgaben -1500 
Anhänge -1509

I Fourier-Reihen. Fourier-Transformation -1509

II $\quad$ Die Diracsche $\delta$-Funktion 1519

III Lagrange- und Hamilton-Mechanik — 1533

Bibliographie - 1551

Sach- und Namenverzeichnis - 1573 
